05,13

\title{
Влияние морфологии на механизмы перемагничивания многослойных тонких пленок Co/Pd
}

\author{
( Ю.В. Касюк ${ }^{1}$, А.А. Максименко ${ }^{1,2}$, Ю.А. Федотова ${ }^{1}$, M. Marszałek ${ }^{2}$, \\ С.К. Лазарук ${ }^{3}$, О.В. Купреева ${ }^{3}$ \\ ${ }^{1}$ Институт ядерных проблем Белорусского государственного университета, \\ Минск, Беларусь \\ ${ }^{2}$ The Henryk Niewodniczański Institute of Nuclear Physics Polish Academy of Sciences, \\ Kraków, Poland \\ ${ }^{3}$ Белорусский государственный университет информатики и радиоэлектроники, \\ Минск, Беларусь \\ E-mail: julia-nechaj@yandex.ru
}

(Поступила в Редакцию 19 апреля 2016 г.)

\begin{abstract}
Методом магнитометрии проведено сравнительное исследование кривых намагниченности сплошной и пористой многослойной пленки $\mathrm{Pd}_{10 n m} /\left[\mathrm{Co}_{0.3 n m} / \mathrm{Pd}_{0.55 n m}\right]_{15} / \mathrm{Pd}_{2 n m}$, осажденной на темплат анодного ТiО 2 . На основании сопоставления зависимостей коэрцитивной силы $H_{C}$ от угла $\theta$ между осью легкого намагничивания и направлением внешнего магнитного поля $H$ с теоретическими зависимостями $H_{C}(\theta)$ для перемагничивания путем движения доменных стенок (по модели Кондорского) и вращения магнитных моментов (по модели Стонера-Вольфарта) выявлены различия в механизмах перемагничивания двух указанных типов пленок. Обсуждается взаимосвязь между различием в морфологии сплошной и пористой пленок и выявленной сменой механизма перемагничивания, а также изменениями значений $H_{C}$ и рассчитанных констант магнитной анизотропии.
\end{abstract}

Работа выполнена в рамках Государственной программы научных исследований „Физическое материаловедение, новые материалы и технологии“(задание 2.44), договора Ф16В2-004 с БРФФИ (Беларусь), гранта 2014/13/N/ST8/00731 National Science Centre (NCN, Poland).

\section{1. Введение}

Тонкие пленки, обладающие перпендикулярной магнитной анизотропией (ПМА), являются востребованными материалами для создания сред перпендикулярной записи информации высокой плотности, а также сенсоров магнитного поля с высоким пространственным разрешением [1]. Экспериментально доказано [1,2], что многослойные пленки $\mathrm{Co} / \mathrm{Pt}$ и $\mathrm{Co} / \mathrm{Pd}$ с толщиной порядка нескольких нанометров проявляют свойство ПМА и характеризуются высокими значениями поля анизотропии $H_{a}$ (порядка нескольких тесла), а также коэрцитивной силы $H_{C}$ в направлении оси легкого намагничивания, т. е. вдоль нормали к плоскости пленок (до 5-7kOe). Причины возникновения ПМА в пленках $\mathrm{Co} / \mathrm{Pt}$ и $\mathrm{Co} / \mathrm{Pd}$ до конца не объяснены. Тем не менее результаты многочисленных исследований данных систем свидетельствуют о высокой способности Со и $\mathrm{Pd}(\mathrm{Pt})$ к формированию сплава, обладающего одноосной ПМА [3]. К числу наиболее вероятных механизмов, отвечающих за формирование ПМА в таком сплаве, относят магнитострикционные эффекты, обусловленные напряжениями, вызванными несоответствием параметров решеток Со и $\mathrm{Pd}$ [3-5]. Также в некоторых работах в качестве источника перпендикулярной анизотропии сплава CoPd называется магнитокристаллическая анизотропия, обусловленная формированием тетрагональной гранецентрированной решетки (так называемой фазы типа $\left.L 1_{0}\right)[1,6]$. Неоднократно показано, что увеличение значений $H_{a}$ и $H_{C}$, характеризующих свойство ПМА многослойных структур $\mathrm{Co} / \mathrm{Pt}$ и $\mathrm{Co} / \mathrm{Pd}$, может быть достигнуто подбором оптимальных толщин слоев, а также их количества $[7,8]$. Соблюдение периодичности слоев $\mathrm{Co}$ и $\mathrm{Pd}(\mathrm{Pt})$ с заданной толщиной, которая, как правило, не превышает 2-5 ев, позволяет формировать на их основе сверхрешетки [9], квазисплавы из перемешанных компонентов Со и $\mathrm{Pd}(\mathrm{Pt})[5]$, которые упорядочиваются в магнитные структуры с ПМА самостоятельно либо под воздействием последующей обработки (отжиг, обучение ионами) $[1,10,11]$. Формирование в таких системах развитых интерфейсов $\mathrm{Co} / \mathrm{Pt}$ и $\mathrm{Co} / \mathrm{Pd}$ помимо усиления ПМА может приводить также к увеличению их намагниченности вследствие спиновой поляризации атомов $\mathrm{Pt}$ и $\operatorname{Pd}[12,13]$.

В подобных анизотропных пленках из сплава (квазисплава) $\mathrm{CoPt}$ и $\mathrm{CoPd}$ магнитные моменты атомов Co ориентируются вдоль нормали пленки, формируя области когерентного намагничивания (магнитные домены). Перемагничивание данных пленок во внешнем магнитном поле происходит по механизму Кондорского [14], который описывает движение доменных стенок. Зависимость $H_{C}$ от ориентации внешнего магнитного поля $H$ для идеальной многодоменной пленки описывается в рамках данной модели соотношением

$$
H_{C}(\theta)=H_{C}(0) / \cos \theta,
$$

где угол $\theta$ задает ориентацию внешнего магнитного поля $H$ относительно оси легкого намагничивания пленки, 
$H_{C}(0)$ - коэрцитивная сила пленки, измеренная вдоль ее оси легкого намагничивания. Следует отметить, что в реальных системах механизм Кондорского наблюдается не во всем диапазоне углов $\theta$. Как правило, в области углов, близких к оси трудного намагничивания $\left(\theta \sim 90^{\circ}\right)$, он сменяется механизмом когерентного вращения магнитных моментов во внешнем поле, описанным Стонером и Вольфартом [15]. Коэрцитивная сила при этом снижается до нулевого значения вдоль оси трудного намагничивания $[15,16]$. Подобная „модификация“ механизма Кондорского дополнительным вкладом когерентного вращения характерна для различных систем (ферромагнитных сплавов $[17,18]$, пленок магнитных сплавов [19,20], многослойных тонких пленок, демонстрирующих ПМА [21], и т.п.). Вклад данных механизмов перемагничивания зависит от многих факторов, среди которых соотношение компонентов системы, морфология пленок и их микроструктура, дефекты и т. п. $[18-20]$.

Нанесение многослойных пленок CoPd на рельефные подложки (полусферы, островки различной формы, пористые темплаты) существенно изменяет их морфологию и приводит к формированию развитого рельефа поверхности [22]. Формирование подобных пористых пленок является перспективной альтернативой существующим технологиям синтеза массивов магнитных наноточек для создания сред записи информации, которая позволяет устранить „суперпарамагнитный предел“ [23]. Кроме того, в пористых пленках увеличивается значение $H_{C}$ за счет дополнительного пиннинга доменных стенок на границах пор [7,24].

В настоящей работе многослойные пленки $\mathrm{Co} / \mathrm{Pd}$ напылялись на пористые подложки анодированного толстостенного $\mathrm{TiO}_{2}$. Основной задачей работы является выявление влияния морфологии исследуемых многослойных пленок $\mathrm{Co} / \mathrm{Pd}$ в результате осаждения на пористые подложки на механизмы их перемагничивания и магнитные характеристики, определяющие величину их ПМА.

\section{2. Объект исследования и методика эксперимента}

Синтез многослойных пленок $\mathrm{Pd}_{10 \mathrm{~nm}} /$ $\left[\mathrm{Co}_{0.3 \mathrm{~nm}} / \mathrm{Pd}_{0.55 n m}\right]_{15} / \mathrm{Pd}_{2 n m}$ (далее $\mathrm{Co} / \mathrm{Pd}$ ) осуществлялся методом термического осаждения в условиях сверхвысокого вакуума с давлением $10^{-7} \mathrm{~Pa}$ на два типа подложек: 1) на пластины $\mathrm{Si} / \mathrm{SiO}_{2}$ для формирования сплошных пленок; 2) на пористый толстостенный $\mathrm{TiO}_{2}$, полученный анодированием фольги Ті. Технология анодирования фольги Ті для получения пористых темплатов $\mathrm{TiO}_{2}$ со средней толщиной стенок около $75 \mathrm{~nm}$ подробно рассмотрена в работах $[25,26]$. Наносимая многослойная пленка содержит буферный слой $\mathrm{Pd}$ толщиной $10 \mathrm{~nm}$, который отделяет последующие слои от подложки, а также защитный слой $\mathrm{Pd}$ толщиной $2 \mathrm{~nm}$. Осаждение

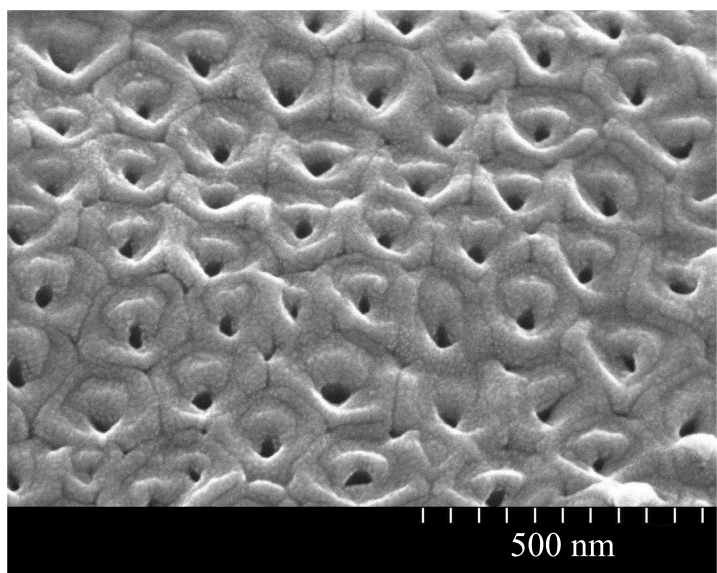

Рис. 1. СЭМ-изображение пленки $\mathrm{Pd} /\left[\mathrm{Co}_{0.3 n m} / \mathrm{Pd}_{0.55 n m}\right] / \mathrm{Pd}$, осажденной на пористый темплат $\mathrm{TiO}_{2}$.

сплошной и пористой пленок осуществлялось в одном экспериментальном цикле для соблюдения одинаковых условий синтеза с целью последующего выявления влияния морфологии на магнитные свойства пленок.

Изображение поверхности пленки $\mathrm{Co} / \mathrm{Pd}$ на пористом темплате $\mathrm{TiO}_{2}$, полученное методом сканирующей электронной микроскопии (СЭМ) (HITACHI S-4800, $15 \mathrm{kV}$ ), приведено на рис. 1. Как видно из этого рисунка, пленка оседает на областях между порами и формирует на поверхности темплата сеть тороидообразных связанных структур, внешний диаметр которых составляет величину порядка $200 \mathrm{~nm}$ [27].

Качество осаждаемых пленок и их структурные параметры (толщина слоев, плотность, шероховатость) для сплошных пленок оценивались методом рентгеновской рефлектометрии на установке Panalyical X'Pert Pro $\left(\lambda_{\mathrm{Cu} K_{\alpha}}=1.54056 \AA\right)$. На основании аппроксимации полученных экспериментальных данных (X'Pert Reflectivity software) установлено, что атомы Со и Рd диффундируют в смежные слои, образуя пленку квазисплава CoPd толщиной $12.7 \mathrm{~nm}$ [28]. Фазовый состав исследуемых пленок анализировался методом рентгеноструктурного анализа на той же экспериментальной установке. Интерпретация соответствующих дифрактограмм подтвердила формирование квазисплава с составом $\mathrm{Co}_{0.4} \mathrm{Pd}_{0.6}$, образующегося из всех осаждаемых атомов Со и части атомов $\mathrm{Pd}$ (за исключением буферного и покрывающего слоев) [28].

Намагниченность $M$ пленок измерялась при комнатной температуре в поле $H$ до $50 \mathrm{kOe}$ на установке MPMS-XL SQUID (Quantum Design). Магнитное поле прикладывалось параллельно $\left(H_{\|}\right)$и перпендикулярно $\left(H_{\perp}\right)$ поверхности пленок. Значения намагниченности пленок $\mathrm{Co} / \mathrm{Pd}$ корректировались на величину парамагнитного (от темплатов $\mathrm{TiO}_{2}$ ) и диамагнитного (от пластин $\left.\mathrm{Si} / \mathrm{SiO}_{2}\right)$ сигналов. Намагниченность рассчитывалась для объема многослойных пленок, не включающего буферный и покрывающий слои $\mathrm{Pd}$. Угловые зависимости коэрцитивной силы $H_{C}$ пленок получены из анализа 
Таблица 1. Значения намагниченности насыщения $M_{S}$, остаточной намагниченности $M_{r}$ и коэрцитивной силы $H_{C}$ сплошной и пористой многослойных пленок $\mathrm{Pd} /\left[\mathrm{Co}_{0.3 n m} / \mathrm{Pd}_{0.55 n m}\right] / \mathrm{Pd}$, полученные из кривых намагниченности $M(H)\left(h_{\text {со }}-\right.$ суммарная толщина слоев $\mathrm{Co}, \mu_{\mathrm{Pd}}-$ магнитный момент атомов $\mathrm{Pd}, \mu_{\mathrm{Pd}} / S-$ удельный магнитный момент атомов Рd единицы площади многослойной пленки)

\begin{tabular}{|c|c|c|c|c|c|c|c|c|c|}
\hline \multirow{2}{*}{ Пленка } & \multirow{2}{*}{$\begin{array}{c}M_{S}, \\
\mathrm{emu} / \mathrm{cm}^{3}\end{array}$} & \multicolumn{2}{|c|}{$M_{r}, \mathrm{emu} / \mathrm{cm}^{3}$} & \multirow{2}{*}{$\begin{array}{c}M_{r} / M_{S} \\
H_{\perp}\end{array}$} & \multirow{2}{*}{$\begin{array}{c}M_{S} h_{\mathrm{Co}}, \\
\mathrm{emu} \cdot \mathrm{cm}^{2}\end{array}$} & \multirow{2}{*}{$\begin{array}{c}\mu_{\mathrm{Pd}} / S, \\
\mathrm{emu} / \mathrm{cm}^{2}\end{array}$} & \multirow{2}{*}{$\begin{array}{c}\mu_{\mathrm{Pd}} \\
\mu_{\mathrm{B}}\end{array}$} & \multicolumn{2}{|c|}{$H_{C}, \mathrm{kOe}$} \\
\hline & & $H_{\|}$ & $H_{\perp}$ & & & & & $H_{\|}$ & $H_{\perp}$ \\
\hline $\begin{array}{l}\text { Сплошная } \\
\text { Пористая }\end{array}$ & $\begin{array}{l}614 \\
628\end{array}$ & $\begin{array}{r}67 \\
130\end{array}$ & $\begin{array}{l}590 \\
446\end{array}$ & $\begin{array}{l}0.96 \\
0.71\end{array}$ & $\begin{array}{l}746 \\
763\end{array}$ & $\begin{array}{r}89 \\
106\end{array}$ & $\begin{array}{l}0.16 \\
0.19\end{array}$ & $\begin{array}{l}0.4 \\
0.5\end{array}$ & $\begin{array}{l}2.2 \\
2.7\end{array}$ \\
\hline
\end{tabular}

зависимостей $M(H)$, измеренных при разных углах $\theta$ направления внешнего магнитного поля $H$ относительно нормали пленок.

\section{3. Результаты и обсуждение}

Кривые намагниченности $M(H)$ многослойной пленки $\mathrm{Co} / \mathrm{Pd}$, осажденной на кремниевую пластину, представлены на рис. 2, $a$ в двух ортогональных ориентациях внешнего магнитного поля $H$ : вдоль нормали пленки $H_{\perp}$
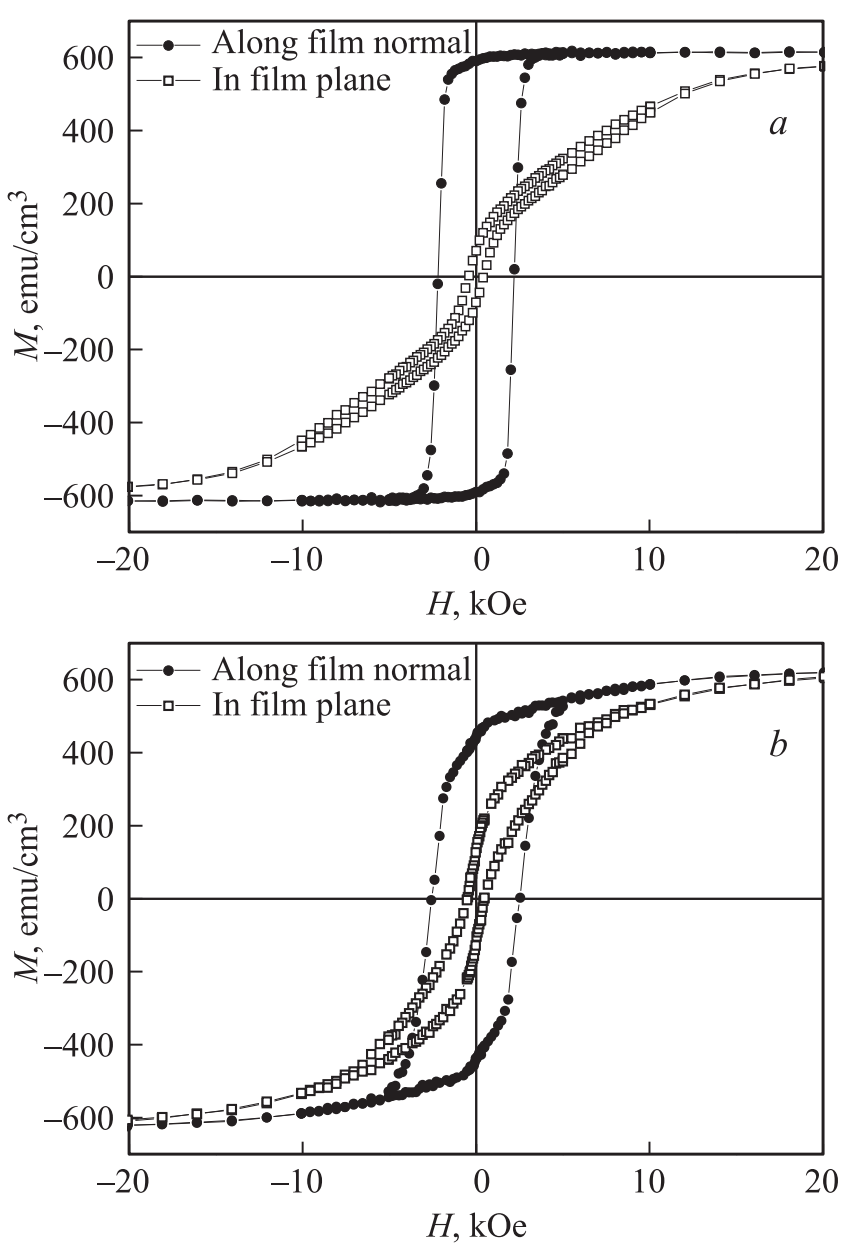

Рис. 2. Кривые намагниченности $M(H)$ сплошной $(a)$ и пористой $(b)$ пленок $\mathrm{Pd} /\left[\mathrm{Co}_{0.3 n m} / \mathrm{Pd}_{0.55 n m}\right] / \mathrm{Pd}$. и вдоль ее поверхности $H_{\|}$. Зависимости $M(H)$ пористой пленки показаны на рис. $2, b$. Значения магнитных характеристик данных пленок (намагниченности насыщения $M_{S}$, остаточной намагниченности $M_{r}$ и коэрцитивной силы $\left.H_{C}\right)$, полученные из данных кривых, приведены в табл. 1.

Как видно из рис. $2 a$, зависимость $M(H)$, измеренная вдоль нормали сплошной пленки $\mathrm{Co} / \mathrm{Pd}$, характеризуется высокими значениями $H_{C}$, а также отношения $M_{r}$ к $M_{S}$ (табл. 1). В то же время кривая намагниченности данной пленки, полученная при приложении внешнего поля вдоль ее поверхности, демонстрирует практически линейный рост до насыщения, а также выход на насыщение при достаточно высоких внешних полях $H$ порядка 20-25kОе. Указанные наблюдения свидетельствуют о том, что сплошная пленка $\mathrm{Co} / \mathrm{Pd}$ характеризуется магнитной анизотропией с ориентацией оси легкого намагничивания вдоль нормали пленки, т. е. перпендикулярной магнитной анизотропией [16]. Высокое поле выхода на насыщение зависимости $M\left(H_{\|}\right)$соответствует значительному полю анизотропии $H_{a}$, характеризующему данную пленку и определяемому как поле, необходимое для ориентации магнитных моментов пленки вдоль ее оси трудного намагничивания [2,16]. Квадратичность $M_{r} / M_{S}$ петли гистерезиса сплошной пленки, близкая к единице в поле $H_{\perp}$ (табл. 1), также указывает на наличие в ней ПМА.

Следует отметить, что пленка $\mathrm{Co} / \mathrm{Pd}$ демонстрирует более высокое значение $M_{S}$ по сравнению с чистым Со, входящим в ее состав. Так, удельное значение магнитного момента изучаемой пленки на единицу ее площади, определяемого произведением $M_{S} h_{\text {Со }}$, где $h_{\text {Со }}-$ суммарная толщина слоев Со (табл. 1), составляет порядка $750 \mathrm{emu} / \mathrm{cm}^{2}$. В то же время единица площади пленки Со той же толщины, что и в составе исследуемого многослойного образца, должна иметь значение намагниченности порядка $530 \mathrm{emu} / \mathrm{cm}^{2}$ при условии, что атомы Со обладают магнитным моментом $1.6 \mu_{\mathrm{B}}$ (как в массивном материале Со) [12]. Ранее показано, что при нанесении монослоев Со на Pt и Pd наблюдается существенное увеличение магнитного момента атомов Со до $2 \mu_{\mathrm{B}}$ [12]. В таком случае монослои Со той же суммарной толщины, что и в исследуемой пленке, могут иметь удельную намагниченность, равную $657 \mathrm{emu} / \mathrm{cm}^{2}$. Таким 
образом, в табл. 1 приведены значения минимального избыточного магнитного момента многослойных пленок $\mu_{\mathrm{Pd}} / S$ по отношению к магнитному моменту атомов Со $\left(2 \mu_{\mathrm{B}}\right)$ в них, который возникает в результате спиновой поляризации атомов $\mathrm{Pd}[12,13]$.

Рассчитанное из представленных экспериментальных кривых $M(H)$ значение магнитных моментов атомов палладия $\mu_{\mathrm{Pd}}$ составляет величину порядка $0.16 \mu_{\mathrm{B}}$ при условии однородной поляризации всех атомов $\mathrm{Pd}$ в пленке. Данное значение находится в соответствии с ранее полученными литературными данными $\left(\mu_{\mathrm{Pd}}=0.23-0.28 \mu_{\mathrm{B}}[12,13]\right)$.

Форма кривой намагниченности $M\left(H_{\perp}\right)$ (рис. 2,b) пористой пленки $\mathrm{Co} / \mathrm{Pd}$ имеет некоторые характерные особенности по сравнению с наблюдаемой для сплошной пленки, проявляющиеся в более плавном выходе намагниченности на насыщение и снижении квадратичности $M_{r} / M_{S}$ петли гистерезиса до 0.71 (табл. 1). Кроме того, кривая $M\left(H_{\|}\right)$показывает более низкое значение поля насыщения (порядка $20 \mathrm{kOe}$ ), характеризующего ПМА, и бо́льшую магнитную восприимчивость $d M / d H$, чем в случае сплошной пленки (рис. 2,a). Таким образом, изменение морфологии пленки в результате ее осаждения на поверхности темплата $\mathrm{TiO}_{2}$ в форме связанных тороидоподобных структур (рис. 1) приводит к ослаблению ПМА по сравнению с характерной для сплошной пленки. Вместе с тем в пористой пленке наблюдается увеличение значения $H_{C}$, измеренного вдоль ее нормали, до $2.7 \mathrm{kOе}$ по сравнению со значением для исходной сплошной пленки $(2.2 \mathrm{kOe})$. Последнее обстоятельство может указывать как на проявление эффекта пиннинга доменных стенок на границах пор при перемагничивании, так и на смену механизма перемагничивания, связанную с подключением вращательных мод, для которых значение $H_{C}$ в случае легкой оси намагничивания является максимальным [16]. Как показано ранее [19], изменение механизма перемагничивания от движения доменных стенок к когерентному вращению в системах, обладающих одноосной магнитной анизотропией, приводит к существенному росту значения $H_{C}$, измеренного вдоль оси легкого намагничивания.

Для исследования возможных механизмов перемагничивания проводилось измерение зависимостей $H_{C}$ сплошных и пористых пленок $\mathrm{Co} / \mathrm{Pd}$ от угла $\theta$ ориентации внешнего поля $H$ относительно их нормали. Полученные зависимости $H_{C}(\theta)$ представлены на рис. 3 . Угол $\theta=0$ соответствует оси легкого намагничивания сплошной пленки $\mathrm{Co} / \mathrm{Pd}$, т. е. ее нормали. При $\theta=90^{\circ}$ внешнее магнитное поле ориентировано вдоль плоскости пленок.

Для сравнения на рис. 3 представлены модельные кривые, полученные для перемагничивания по механизму Кондорского [14,17,18] и Стонера-Вольфарта [29].

Модифицированная модель Кондорского, описывающая движение доменных стенок, с учетом вклада когерентного вращения магнитных моментов в области

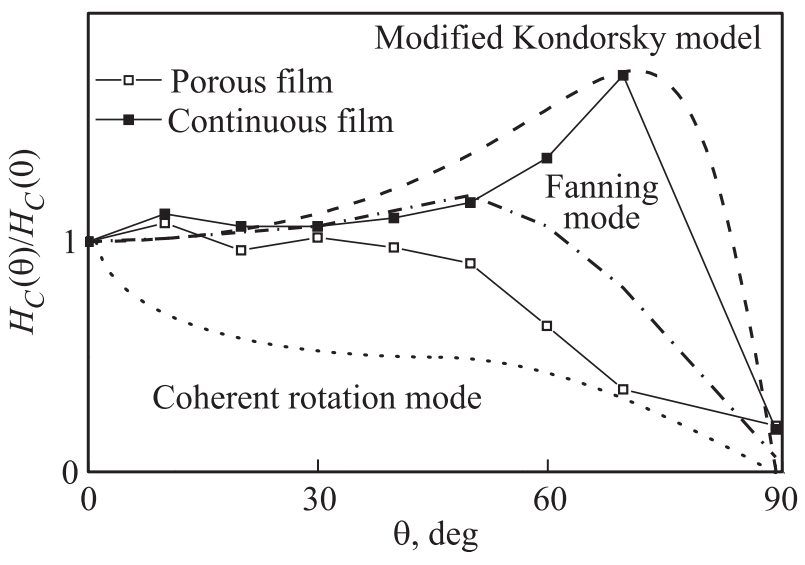

Рис. 3. Зависимости $H_{C}(\theta)$ сплошной и пористой пленок $\mathrm{Pd} /\left[\mathrm{Co}_{0.3 n m} / \mathrm{Pd}_{0.55 n m}\right] / \mathrm{Pd}$ вместе с модельными кривыми, описывающими перемагничивание по модифицированному механизму Кондорского, механизмам когерентного (coherent rotation mode) и некогерентного (fanning mode) вращения магнитных моментов.

больших углов $\theta$ предполагает, что зависимость $H_{C}(\theta)$ может быть описана выражением $[18,20]$

$$
H_{C}(\theta) / H_{C}(0)=\frac{\left(N_{x}+N_{N}\right) \cos \theta}{N_{z} \sin ^{2} \theta+\left(N_{x}+N_{N}\right) \cos ^{2} \theta},
$$

где параметр $y=\left(N_{x}+N_{N} / N_{z}\right)$ определяет соотношение между ПМА пленки и размагничивающими эффектами, обусловленными магнитостатической энергией, характерной для тонких пленок; $N_{x}$ и $N_{z}$ - размагничивающие факторы пленки в двух ортогональных ориентациях, а $N_{N}=H_{a} / M_{S}-$ формальный параметр, подобный размагничивающему фактору и характеризующий анизотропию, отличную от анизотропии формы пленки (например, магнитокристаллическую).

Показанная на рис. 3 модельная кривая, описывающая механизм Кондорского, получена из соотношения (2). Наилучшее соответствие экспериментальным данным, описывающим сплошную пленку $\mathrm{Co} / \mathrm{Pd}$, наблюдается при значении параметра $y=8$. На основании данного значения может быть оценена величина поля магнитокристаллической анизотропии данной пленки с учетом того, что пленка представляет собой квазиоднородный ферромагнитный сплав, т.е. ее размагничивающие факторы вдоль поверхности пленки равны нулю, а вдоль ее нормали $N_{z}=1$. Данная оценка приводит к величине поля $H_{a}$ порядка $60 \mathrm{kOe}$, что существенно выше, чем значение $H_{a} \sim 20-25 \mathrm{kOe}$, оцененное по экспериментальной кривой намагниченности $M(H)$ данной пленки, измеренной вдоль ее поверхности, т.е. вдоль оси трудного намагничивания (рис. 2,a). Подобное расхождение, вероятно, обусловлено грубой оценкой $H_{a}$ обоими методами вследствие недостаточно хорошего соответствия экспериментальной кривой $H_{C}(\theta)$ теоретической зависимости для перемагничивания по механизму Кондорского, а также вследствие плавного выхода зависимости $M(H)$ 
на насыщение (рис. 2,a) вместо характеризующего $H_{a}$ излома зависимости в одной точке, соответствующей конкретному полю насыщения, что усложняет задачу определения данного параметра.

Характер зависимости $H_{C}(\theta)$, полученной для пористой пленки $\mathrm{Co} / \mathrm{Pd}$, заметно отличается от рассмотренной выше кривой для сплошной пленки. Отсутствие роста $H_{C}$ с увеличением угла $\theta$, характерного для механизма Кондорского, а также выраженного максимума в области высоких значений $\theta$, типичного для модифицированного механизма Кондорского, позволяет предположить, что движение доменных стенок не является основным механизмом перемагничивания в пористом образце $\mathrm{Co} / \mathrm{Pd}$. Форма зависимости $H_{C}(\theta)$ данной пленки скорее соответствует теоретическим кривым, характерным для перемагничивания посредством вращения магнитных моментов. Для сравнения на рис. 3 приведены теоретические зависимости для механизмов когерентного вращения магнитных моментов (coherent rotation) и некогерентного вращения (fanning), которые различаются в области малых углов $\theta<45^{\circ}$. Хорошее согласие экспериментальной кривой и модельной зависимости, соответствующей механизму некогерентного вращения, вероятно, обусловлено формированием сложных разупорядоченных конфигураций магнитных моментов в образуемых на поверхности пленки тороидообразных структурах. Сложная морфология пористой пленки и доминирование вращательных мод в процессах ее перемагничивания позволяют рассматривать данный образец не как многодоменную пленку, а как систему магнитных наноструктур, перемагничивание каждой из которых определяет механизм перемагничивания системы в целом.

Кривая намагниченности $M\left(H_{\perp}\right)$ такой системы аппроксимировалась в рамках модели когерентного вращения магнитных моментов, разработанной Стонером и Вольфартом [15]. Модель когерентного вращения является наиболее простой и универсальной. Она успешно применялась для большого числа систем, демонстрирующих одноосную магнитную анизотропию [23,30], в том числе ее применение расширено на магнитные структуры, связанные магнитостатическим (диполь-дипольным) взаимодействием [31,32], а также на однородные сплошные [33-36] и перфорированные [37,38] пленки с одноосной магнитной анизотропией.

Кривая намагниченности $M\left(H_{\perp}\right)$ пористой пленки $\mathrm{Co} / \mathrm{Pd}$ вместе с аппроксимирующей ее модельной кривой намагничивания по механизму Стонера-Вольфарта, приведена на рис. 4.

Теоретическая кривая $\cos \varphi(h)$, построенная в относительных координатах, является решением уравнения $[15,16]$

$$
h \sin \varphi+\frac{1}{2} \sin 2(\varphi-\theta)=0,
$$

где $h=H / H_{\text {eff }}, H_{\text {eff }}-$ поле эффективной магнитной анизотропии; $\varphi$ - угол между магнитным моментом и внешним магнитным полем $H ; \theta$ - угол между осью легкого намагничивания и внешним магнитным полем $H$. Эффективная магнитная анизотропия пленки, как упоминалось ранее, включает ПМА, обусловленную одноосной анизотропией сплава $\mathrm{CoPd}$, а также магнитостатическую анизотропию, вызванную высоким размагничивающим фактором квазиоднородной ферромагнитной пленки вдоль ее нормали [16,39]. Поэтому поле эффективной магнитной анизотропии $H_{\text {eff }}$ содержит вклады от поля магнитокристаллической анизотропии $H_{a}$ и размагничивающего поля $H_{d}$. В направлении нормали пленки $H_{\mathrm{eff}}=H_{a}-4 \pi M_{S}$, поскольку $H_{d}=-4 \pi M_{S}[2,16]$. Рассчитанные значения $H_{d}$ суммированы для многослойных пленок в табл. 2.

Аппроксимация экспериментальной кривой намагниченности $M\left(H_{\perp}\right)$ соотношением (2) позволяет оценить значения параметра $\theta=\arccos \left(M_{r} / M_{S}\right)$, определяющего положение оси легкого намагничивания пленок относительно их нормали, и $H_{\text {eff. }}$ Оба указанных параметра описывают эффективную магнитную анизотропию пленок и приведены в табл. 2. Представленная на рис. 4 аппроксимация хорошо коррелирует с экспериментальными данными для пористой пленки $\mathrm{Co} / \mathrm{Pd}$, что подтверждает возможность описания перемагничивания в ней в соответствии с вращательным механизмом и указывает на применимость к данной пленке модели когерентного вращения магнитных моментов. Наблюдаемые отклонения экспериментальной и аппроксимирующей зависимостей в области полей переключения ( $3 \mathrm{kOe})$, когда происходит изменение направления магнитных моментов относительно оси легкого намагничивания на противоположное, свидетельствуют о дисперсии осей легкого намагничивания в пленке, обусловленной ее сложным рельефом.

Полученное из аппроксимации поле $H_{\text {eff }}$ составляет порядка $6 \mathrm{kOe}$, что соответствует константе эффектив-

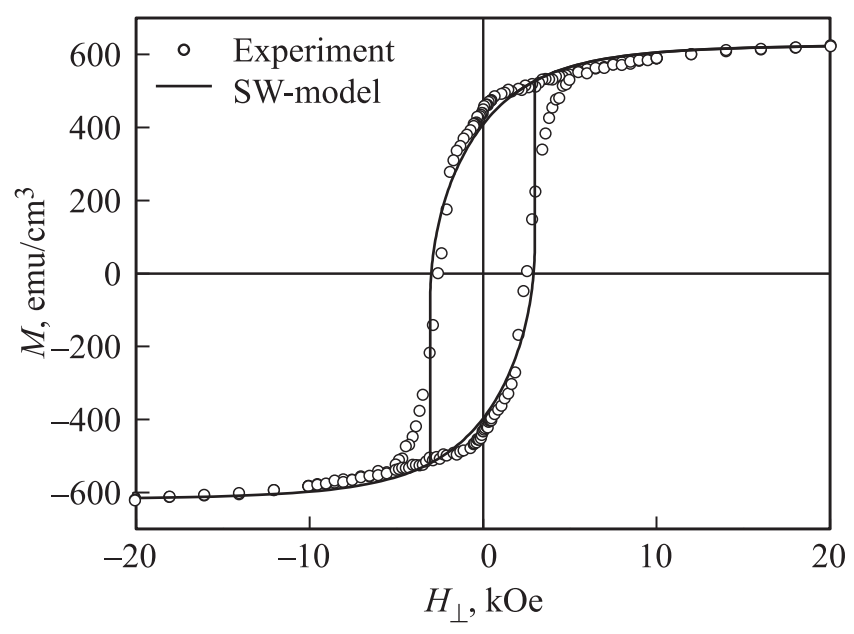

Рис. 4. Зависимость $M\left(H_{\perp}\right)$ пористой пленки $\mathrm{Pd} /\left[\mathrm{Co}_{0.3 n m} / \mathrm{Pd}_{0.55 n m}\right] / \mathrm{Pd}$ вместе с аппроксимирующей модельной кривой перемагничивания по механизму Стонера-Вольфарта (SW-model). 
Таблица 2. Значения поля $H_{\text {eff }}$ и константы эффективной магнитной анизотропии $K_{\text {eff }}$ сплошной и пористой многослойных пленок $\mathrm{Pd} /\left[\mathrm{Co}_{0.3 n m} / \mathrm{Pd}_{0.55 n m}\right] / \mathrm{Pd}$, оцененные с помощью аппроксимации их зависимостей $M\left(H_{\perp}\right)$ на основании модели Стонера-Вольфарта [15] и на основе оценки энергии магнитной анизотропии по разности площадей между кривыми намагниченности $M_{\left(H_{\|}\right)}$и $M\left(H_{\perp}\right)$ и осью $M[16,40]\left(H_{a}\right.$ и $K_{U}-$ поле и константа одноосной перпендикулярной анизотропии, $H_{d}-$ размагничивающее поле пленки вдоль ее нормали, $\left.\theta=\arccos \left(M_{r} / M_{S}\right)\right)$

\begin{tabular}{|c|c|c|c|c|c|c|c|c|}
\hline \multirow{2}{*}{ Пленка } & \multicolumn{2}{|c|}{$\begin{array}{c}\text { Модель } \\
\text { Стонера-Вольфарта }\end{array}$} & \multicolumn{2}{|c|}{$\begin{array}{c}\text { Метод разности } \\
\text { площадей }\end{array}$} & \multirow{2}{*}{$\begin{array}{l}H_{d} \\
\mathrm{kOe}\end{array}$} & \multirow{2}{*}{$\theta,^{\circ}$} & \multirow{2}{*}{$\begin{array}{l}H_{a} \\
\mathrm{kOe}\end{array}$} & \multirow{2}{*}{$\begin{array}{c}K_{U} \cdot 10^{-6} \\
\mathrm{erg} / \mathrm{cm}^{3}\end{array}$} \\
\hline & $\begin{array}{l}H_{\text {eff }} \\
\mathrm{kOe}\end{array}$ & $\begin{array}{l}K_{\mathrm{eff}} \cdot 10^{-6} \\
\mathrm{erg} / \mathrm{cm}^{3}\end{array}$ & $\begin{array}{l}H_{\text {eff }}, \\
\mathrm{kOe}\end{array}$ & $\begin{array}{c}K_{\text {eff }} \cdot 10^{-6} \\
\mathrm{erg} / \mathrm{cm}^{3}\end{array}$ & & & & \\
\hline $\begin{array}{l}\text { Сплошная } \\
\text { Пористая }\end{array}$ & $\frac{-}{6.0}$ & $\begin{array}{l}- \\
1.9\end{array}$ & $\begin{array}{r}10.4 \\
4.8\end{array}$ & $\begin{array}{l}3.2 \\
1.5\end{array}$ & $\begin{array}{l}7.7 \\
-\end{array}$ & $\begin{array}{l}16 \\
50\end{array}$ & $\begin{array}{c}18.1 \\
-\end{array}$ & $\begin{array}{l}5.6 \\
-\end{array}$ \\
\hline
\end{tabular}

ной магнитной анизотропии $H_{\mathrm{eff}} M_{S} / 2=1.9 \cdot 10^{6} \mathrm{erg} / \mathrm{cm}^{3}$ (табл. 2). Это значение коррелирует с полученным ранее для подобных многослойных систем [40,41]. Оцененный с помощью аппроксимации угол $\theta$ ориентации оси легкого намагничивания для данной пленки принимает довольно высокое значение $50^{\circ}$, что близко к углу $\theta=45^{\circ}$, характеризующему изотропные системы. Тем не менее исследуемая пористая пленка не является изотропной. Так, из рис. $2, b$ очевидно, что площадь под кривой $M(H)$ значительно больше площади, покрываемой $M\left(H_{\|}\right)[16,40]$. Высокое значение угла $\theta$ может быть объяснено следующим образом. Эффективная магнитная анизотропия исследуемых пленок определяется, как упоминалось ранее, конкуренцией энергий одноосной перпендикулярной (магнитострикционной либо магнитокристаллической) и магнитостатической анизотропии. В случае сплошной пленки оси легкого намагничивания каждого из перечисленных типов магнитной анизотропии ориентированы практически ортогонально друг к другу. При этом результирующая ось эффективной магнитной анизотропии ориентирована вдоль легкой оси более сильной анизотропии (с более высокими значениями $H_{a}$ и $K$ ) [16]. В случае сплошной многослойной пленки результирующая ось эффективной магнитной анизотропии ориентирована вдоль ее нормали, поскольку поле ее магнитостатической анизотропии $H_{d}$ составляет всего $7.7 \mathrm{kOe}$ (табл. 2), тогда как поле ее ПМА превышает $20 \mathrm{kOe}$ (из оценки выхода на насыщение зависимости $M\left(H_{\|}\right)$) либо $\sim 60 \mathrm{kOe}$ (в соответствии с аппроксимацией зависимости $H_{C}(\theta)$ согласно модифицированной модели Кондорского).

В случае же пористой пленки вследствие ее сложной морфологии может наблюдаться дисперсия осей перпендикулярной анизотропии различных областей образца. В этом случае оси перпендикулярной и магнитостатической анизотропии не направлены больше ортогонально друг другу, вследствие чего формируется их равнодействующая [16], ориентированная между данными осями. Это и приводит к возрастанию угла $\theta$. Данное обстоятельство также, вероятно, обусловливает смену механизма перемагничивания в пористой пленке с движения доменных стенок, характеризующего сплошную пленку, на вращение магнитных моментов.

Оценка параметров магнитной анизотропии сплошной пленки $\mathrm{Co} / \mathrm{Pd}$ описанным выше способом (модель Стонера-Вольфарта) не является корректной, поскольку в соответствии с зависимостью $H_{C}(\theta)$ перемагничивание в ней происходит преимущественно по механизму движения доменных стенок. Поэтому для определения соответствующего значения константы ее эффективной магнитной анизотропии $K_{\text {eff }}$ может быть применен общепринятый метод расчета энергии магнитной анизотропии по площади между кривыми намагниченности в двух ортогональных ориентациях внешнего магнитного

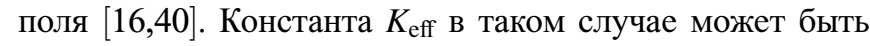
оценена как

$$
K_{\text {eff }}=\int_{0 \_ \text {in }}^{M_{S}} H d M-\int_{0 \_ \text {out }}^{M_{S}} H d M,
$$

где первый интеграл соответствует ориентации внешнего поля вдоль плоскости пленки (in), а второй — вдоль eе нормали (out). Полученные значения $K_{\text {eff }}$ приведены в табл. 2 вместе с рассчитанными из них значениями $H_{\text {eff }}=2 K_{\text {eff }} / M_{S}$ для сплошной и пористой пленок.

Следует отметить, что оба применяемых метода оценки позволяют получить близкие значения $H_{\text {eff }}$ и $K_{\text {eff }}$ для пористого образца, что подтверждает достоверность полученных значений и установленного механизма его перемагничивания путем вращения магнитных моментов.

Для сплошной пленки на основании рассчитанных значений $H_{\mathrm{eff}}$ и $K_{\mathrm{eff}}$ с помощью соотношения $H_{\text {eff }}=H_{a}-4 \pi M_{S}$ представляется возможным оценить величину ее поля одноосной перпендикулярной анизотропии $H_{a}$, которая составляет $18.1 \mathrm{kOe}$ (табл. 2). Это значение близко к оцененному по рис. 2, $a$ полю выхода на насыщение зависимости $M\left(H_{\|}\right)$данной пленки. Полученному полю анизотропии соответствует константа одноосной анизотропии $K_{U}=5.6 \cdot 10^{6} \mathrm{erg} / \mathrm{cm}^{3}$, значение которой близко к соответствующему параметру перпендикулярной анизотропии системы $\mathrm{Co} / \mathrm{Pd}[2,9]$. 
Подобный расчет $K_{U}$ не может быть корректно выполнен для пористой пленки, поскольку сложность ее морфологии не позволяет рассчитать для нее размагничивающие факторы в разных направлениях, а следовательно, и размагничивающее поле $H_{d}$.

\section{4. Заключение}

Комплексный анализ кривых намагниченности $M(H)$ и угловых зависимостей коэрцитивной силы $H_{C}(\theta)$ многослойных пленок $\mathrm{Pd} /\left[\mathrm{Co}_{0.3 n m} / \mathrm{Pd}_{0.55 n m}\right] / \mathrm{Pd}$, нанесенных на плоские и пористые подложки, позволяет установить механизмы их перемагничивания во внешнем поле.

Сплошные пленки $\mathrm{Pd} /\left[\mathrm{Co}_{0.3 n m} / \mathrm{Pd}_{0.55 n m}\right] / \mathrm{Pd}$, осажденные на плоские пластины $\mathrm{Si} / \mathrm{SiO}_{2}$, демонстрируют ПМА с полем анизотропии $H_{a} \sim 18 \mathrm{kOe}$ и константой $K_{U}=5.6 \cdot 10^{6} \mathrm{erg} / \mathrm{cm}^{3}$. Размагничивающие эффекты, связанные с формой пленок, приводят к ослаблению их ПМА, которая характеризуется эффективными параметрами $H_{\text {eff }} \sim 11 \mathrm{kOe}$ и $K_{\text {eff }}=3.2 \cdot 10^{6} \mathrm{erg} / \mathrm{cm}^{3}$, оцененными по площади, ограничиваемой кривыми $M\left(H_{\perp}\right)$ и $M\left(H_{\|}\right)$. Как следует из зависимостей $H_{C}(\theta)$, перемагничивание сплошных пленок осуществляется путем движения доменных стенок в соответствии с моделью Кондорского, модифицированной вкладом когерентного вращения магнитных моментов в области высоких значений углов $\theta$ ориентации внешнего поля относительно нормали пленок.

В случае пористых пленок $\mathrm{Pd} /\left[\mathrm{Co}_{0.3 n m} / \mathrm{Pd}_{0.55 n m}\right] / \mathrm{Pd}$ их морфология в виде сети торообразных связанных структур приводит к изменению механизма перемагничивания, которое осуществляется вращением магнитных моментов во внешнем поле. Смена механизма перемагничивания подтверждается характером зависимости $H_{C}(\theta)$ данных пленок и хорошим согласием между экспериментальными и модельными кривыми намагниченности $M(H)$, описывающими перемагничивание путем когерентного вращения магнитных моментов по модели Стонера-Вольфарта. Оценка эффективных параметров магнитной анизотропии данных пленок в рамках модели Стонера-Вольфарта позволяет получить значения $H_{\text {eff }} \sim 6 \mathrm{kOe}$ и $K_{\text {eff }}=1.9 \cdot 10^{6} \mathrm{erg} / \mathrm{cm}^{3}$, что в 2 раза ниже, чем в случае сплошных пленок. Вместе с тем в пористых пленках наблюдается увеличение величины $H_{C}$ до $2.7 \mathrm{kOe}$ по сравнению со значением для сплошных пленок (2.2 kOe) вследствие смены механизма перемагничивания и пиннинга магнитных моментов на нанопорах.

Снижение эффективных параметров, характеризующих перпендикулярную магнитную анизотропию $\left(H_{\text {eff }}\right.$ и $\left.K_{\text {eff }}\right)$ многослойных пленок $\mathrm{Pd} /\left[\mathrm{Co}_{0.3 n m} / \mathrm{Pd}_{0.55 n m}\right] / \mathrm{Pd}$, a также изменение механизма их перемагничивания вызвано дисперсией ориентации осей перпендикулярной анизотропии сплава $\mathrm{CoPd}$, характеризующей различные области пленки, в результате усложнения ее морфологии и формирования развитого рельефа в виде связанных торообразных структур.

\section{Список литературы}

[1] J.H. Judy. J. Magn. Magn. Mater. 287, 16 (2004).

[2] Z. Liu, R. Brandt, O. Hellwig, S. Flore, T. Thomson, B. Terris, H. Schmidt. J. Magn. Magn. Mater. 323, 1623 (2011).

[3] J. Carrey, E.A. Berkowitz, W.F. Egelhoff,jr., D.J. Smith. Appl. Phys. Lett. 83, 5259 (2003).

[4] S.-K. Kim, Y.-M. Koo, V.A. Chernov. J.Phys. IV (France) 7, C2, C2-1097 (1997).

[5] S.-K. Kim, S.-C. Shin. J. Appl. Phys. 89, 3055 (2001).

[6] Е.М. Артемьев, Л.В. Живаева. ЖТФ 78, 10, 129 (2008).

[7] O. Hellwig, T. Hauet, T. Thomson, E. Dobisz, J.D. RisnerJamtgaard, D. Yaney, B.D. Terris, E.E. Fullerton. Appl. Phys. Lett. 95, 232505 (2009).

[8] B. Hu, N. Amos, Y. Tian, J. Butler, D. Litvinov, S. Khizroev. J. Appl. Phys. 109, 034314 (2011).

[9] K. Yakushiji, T. Saruya, H. Kubota, A. Fukushima, T. Nagahama, S. Yuasa, K. Ando. Appl. Phys. Lett. 97, 232508 (2010).

[10] H. Bernas, J.-Ph. Attané, K.-H. Heinig, D. Halley, D. Ravelosona, A. Marty, P. Auric, C. Chappert, Y. Samson. Phys. Rev. Lett. 91, 077203 (2003).

[11] M.S. Martín-González, F. Briones, J.M. García-Martín, J. Montserrat, L. Vila, G. Faini, A.M. Testa, D. Fiorani, H. Rohrmann. J. Magn. Magn. Mater. 322, 2762 (2010).

[12] G. Moulas, A. Lehnert, S. Rusponi, J. Zabloudil, C. Etz, S. Ouazi, M. Etzkorn, P. Bencok, P. Gambardella, P. Weinberger, H. Brune. Phys. Rev. B 78, 214424 (2008).

[13] I. Galanakis. J. Magn. Magn. Mater. 377, 291 (2015).

[14] E. Kondorski. J. Phys. (USSR) 2, 161 (1940).

[15] E.C. Stoner, E.P. Wohlfarth. Phil. Trans. Roy. Soc. A 240, 599 (1948).

[16] B.D. Cullity, C.D. Graham. Introduction to magnetic materials. 2nd ed. Wiley-IEEE Press, Hoboken, N.J. (2009). 568 p.

[17] P. Pawlik, J. Wyslocki, W. Kaszuwara, M. Leonowicz. J. Magn. Magn. Mater. 242-245, 1344 (2002).

[18] N.P. Suponev, R.M. Grechishkin, M.B. Lyakhova, Yu.E. Pushkar. J. Magn. Magn. Mater. 157-158, 376 (1996).

[19] T.R. Gao, S.P. Hao, P.M. Zhou, L. Sun. J. Appl. Phys. 100, 073909 (2006).

[20] M. Mathews, E.P. Houwman, H. Boschker, G. Rijnders, D.H.A. Blank. J. Appl. Phys. 107, 013904 (2010).

[21] J. Zhang, Y. Li, F. Wang, B. Shen, J. Sun. J. Appl. Phys. 107, 043911 (2010).

[22] Y.X. Wang, W. Li, Y.J. Zhang, Y. Liu, S.S. Liu, X.L. Zhang, Y.X. Jiang, J.H. Yang. J. Appl. Phys. 113, 163901 (2013).

[23] R. Dittrich, G. Hu, T. Schrefl, T. Thomson, D. Suess, B.D. Terris, J. Fidler. J. Appl. Phys. 97, $10 J 705$ (2005).

[24] J. Sort, B. Dieny, M. Fraune, C. Koenig, F. Lunnebeach, B. Beschoten, G. Guntherodt. Appl. Phys. Lett. 84, 3696 (2004).

[25] K. Lazarouk, D.A. Sasinovich, O.V. Kupreeva, T.I. Orehovskaia, N. Rochdi, F. Arnaud d'Avitaya, V.E. Borisenko. Thin Solid Films 526, 41 (2012).

[26] S.K. Lazarouk, D.A. Sasinovich, V.E. Borisenko, A. Muravski, V. Chigrinov, H.S. Kwok. J. Appl. Phys. 107, 033527 (2010). 
[27] A. Maximenko, J. Fedotova, M. Marszalek, O. Kupreeva, S. Zavadski. In: Physics, chemistry and application of nanostructures / Eds V. Borisenko, S. Gaponenko, V. Gurin, C. Kam. World Scientific, Singapore (2015). P. 132.

[28] A. Maximenko, J. Fedotova, M. Marszalek, A. Zarzycki, Y. Zabila. J. Magn. Magn. Mater. 400, 200 (2015).

[29] G.A. Milne, D.J. Dunlop. J. Geophys. Res. 111, B12S08 (2006).

[30] S.M. Ryabchenko, A.A. Timopheev, V.M. Kalita, O.V. Stognei, A.V. Sitnikov. J. Appl. Phys. 109, 043903 (2011).

[31] E.P. Wohlfarth. Proc. Roy. Soc. Lond. A 232, 208 (1955).

[32] A.A. Timopheev, S.M. Ryabchenko, V.M. Kalita, A.F. Lozenko, P.A. Trotsenko, V.A. Stephanovich, A.M. Grishin, M. Munakata. J. Appl. Phys. 105, 083905 (2009).

[33] D. Spenato, V. Castel, S.P. Pogossian, D.T. Dekadjevi, J.B. Youssef. Appl. Phys. Lett. 91, 062515 (2007).

[34] J. Camarero, J. Sort, A. Hoffmann, J.M. Garcia-Martın, B. Dieny, R. Miranda, J. Nogues. Phys. Rev. Lett. 95, 057204 (2005).

[35] Ch. Binek, A. Hochstrat, W. Kleemann. J. Magn. Magn. Mater. 234, 353 (2001).

[36] M. Volmer, J. Neamtu. Romanian Rep. Phys. 56, 367 (2004).

[37] A. Hoffmann, M. Grimsditch, J.E. Pearson, J. Nogues, W.A.A. Macedo, I.K. Schuller. Phys. Rev. B 67, 220406 (2003).

[38] S.H. Chung, A. Hoffmann, M. Grimsditch. Phys. Rev. B 71, 214430 (2005).

[39] M.T. Johnson, P.J.H. Bloemen, F.J.A. den Broeder, J.J. de Vries. Rep. Prog. Phys. 59, 1409 (1996).

[40] D. Navas, M. Hernandez-Velez, M. Vazquez, W. Lee, K. Nielsch. Appl. Phys. Lett. 90, 192501 (2007).

[41] J.I. Hong, S. Sankar, A.E. Berkowitz, W.F. Egelhoff,jr. J. Magn. Magn. Mater. 285, 359 (2005) 